\title{
ON CLOSED STARSHAPED SETS AND BAIRE CATEGORY
}

\author{
GERALD BEER
}

\begin{abstract}
Let $C$ be a closed set of second category in a normed linear space, and let $C^{*}$ be the subset of $C$ each point of which sees all points of $C$ except a set of first category. If $C^{*}$ is nonempty, then $C^{*}$ is a closed convex set. Moreover, $C=K \cup P$ where $K$ is a closed starshaped set with convex kernel $C^{*}$ and $P$ is a set of first category.
\end{abstract}

Let $C$ be a set in a linear space. If $\{x, y\} \subset C$ and the line segment joining $x$ to $y$ lies in $C$, then we say that $x$ sees $y$ via $C$. The set of points that $x$ sees via $C$ is called the star of $x$ (relative to $C$ ). If the star of some point $x$ in $C$ is the entire set, then we say that $C$ is starshaped with respect to $x$, and we call the set of points that see each point of $C$ the convex kernel of $C$. If the linear space is equipped with a norm, then we can talk about points in $C$ that see essentially all of $C$ in a topological sense. It is the purpose of this article to characterize closed sets in a normed linear space that have such points.

Before proceeding, we set forth some notation and additional terminology. If $x$ is a vector in a normed linear space $L$, then $B_{\lambda}(x)$ will denote the open ball with radius $\lambda$ and center $x$. If $y \neq x$, then $\operatorname{seg}[x, y]$ will symbolize the closed line segment joining the two points. If $C \subset L$ and $x$ is in $C$, then $S(x)$ will denote the star of $x$. A subset $C$ of a topological space is called nowhere dense if its closure has empty interior. It is called a set of first category if and only if it can be expressed as a countable union of nowhere dense sets; otherwise, it is called a set of second category. If the relative complement of a subset $K$ of $C$ is of first category, then $K$ is called a residual subset of $C$. If $C$ is a subset of a normed linear space, then $C^{*}$ will denote those points in $C$ whose star is a residual subset of $C$. Thus, if $C$ is of second category, then $C^{*}$ is that subset of $C$ whose points see all but an insignificant set of points in a topological sense.

LEMMA 1. Let $L$ be a normed linear space. If $x \in C \subset L$ and the star $S(x)$ of $x$ relative to $C$ is a set of second category, then $S(x) \cap B_{\lambda}(x)$ is a set of second category for each positive $\lambda$.

Proof. Without loss of generality we can assume that $\lambda<1$. We can represent $S(x)$ as follows:

$$
S(x)=\bigcup_{n=1}^{\infty} B_{n}(x) \cap S(x) .
$$

Received by the editors September 27, 1978 and, in revised form, October 12, 1978.

AMS (MOS) subject classifications (1970). Primary 52A30; Secondary 54C50. 
Since the sets of first category form a $\sigma$-ideal of sets, there exists $n$ such that $E=B_{n}(x) \cap S(x)$ is of second category. Hence, the image of $E$ under the homeomorphism $g(z)=x+(\lambda / n)(z-x)$ is a subset of $B_{\lambda}(x)$ of second category. Morever, since $\lambda / n<1$, we have $g(E) \subset S(x)$. Hence, $S(x) \cap B_{\lambda}(x)$ is of second category.

Corollary. Let $C$ be a closed set in a normed linear space. If $C^{*}$ is nonempty, then the star of each point in $C^{*}$ includes $\{x \in C: S(x)$ is of second category $\}$.

Proof. Let $y \in C^{*}$ be arbitrary. If $S(x)$ is of second category, Lemma 1 implies that $y$ sees points in each neighborhood of $x$. Thus, $x$ is in $S(y)$ because $S(y)$ is a closed set.

The previous corollary serves to trivially characterize those closed starshaped sets $C$ in a normed linear space for which the star of each point is of second category: $C^{*}$, the set of points in $C$ whose star is a residual subset of $C$, is nonempty. Notice also that since $C^{*}$ will be the convex kernel of such a set $C$, it must necessarily be a closed convex set [4]. But what if some points of $C$ see only a set of first category via $C$ ? If $C^{*}$ is nonempty, is the set still closed and convex, and how is $C$ related to a starshaped set? It is the purpose of this article to answer these questions.

Our major tools will be variants of two basic theorems in category theory [3]: a polar form of the Kuratowski-Ulam Theorem and a general form of the Banach Category Theorem.

THEOREM 1. Let $L$ be a normed linear space and let $E$ be a subset of first category. For each $x$ of norm one, let $E_{x}=\{\alpha: \alpha>0$ and $\alpha x \in E\}$. Then $\left\{x: E_{x}\right.$ is of second category in $R\}$ is a subset of first category in the relative topology for $\Delta=\{x$ : $\|x\|=1\}$.

Proof. Since the assignment $E \rightarrow E_{x}$ preserves set operations, it suffices to show that if $E$ is a nowhere dense closed set, then $E_{x}$ is nowhere dense for all $x$ except a set of first category in $\Delta$. Denote the dense open set $L-E$ by $G$, and let $\left\{V_{n}\right.$ : $\left.n \in Z^{+}\right\}$be a countable collection of intervals that serve as a base for the usual topology on $(0, \infty)$. For each positive integer $n$ define $G_{n}$ as follows:

$$
G_{n}=\left\{x: \alpha x \in G \text { for some } \alpha \text { in } V_{n}\right\} \cap \Delta .
$$

We first show that $G_{n}$ is open in the relative topology. To see this, fix $x_{0}$ in $G_{n}$ and choose $\alpha_{0}$ in $V_{n}$ such that $\alpha_{0} x_{0} \in G$. By the continuity of the map $f(x)=\alpha_{0} x$ at $x=x_{0}$, there exists a neighborhood $W$ of $x_{0}$ such that $f(W) \subset G$. Thus, $W \cap \Delta \subset$ $G_{n}$, and $G_{n}$ is open.

We next verify that $G_{n}$ is dense in the relative topology. Let $U$ be an open set in $L$ that meets $\Delta$. We must show that $G_{n} \cap U \neq \varnothing$. Define a subset $T$ of $L$ as follows:

$$
T=\left\{\alpha x: x \in U \cap \Delta \text { and } \alpha \in V_{n}\right\} .
$$

If we could show that $T$ contains an open set, then $T \cap G \neq \varnothing$ whence $U \cap G_{n} \neq$ $\varnothing$. We shall show that $T$ is actually open. To this end fix $x_{0}$ in $U \cap \Delta$ and $\alpha_{0}$ in $V_{n}$. Suppose for each positive integer $n$ we have $B_{1 / n}\left(\alpha_{0} x_{0}\right) \cap \tilde{T} \neq \varnothing$. For each $n$ 
choose $x_{n}$ in $B_{1 / n}\left(\alpha_{0} x_{0}\right) \cap \tilde{T}$. Since $x_{n} /\left\|x_{n}\right\|$ is in $U$ eventually, it follows that $\left\|x_{n}\right\|$ is not in $V_{n}$ for all $n$ sufficiently large. Since this contradicts $\lim _{n \rightarrow \infty}\left\|x_{n}\right\|=\left\|\alpha_{0} x_{0}\right\|$ $=\alpha_{0}$, there exists a neighborhood of $\alpha_{0} x_{0}$ contained in $T$. From the above remarks it follows that $G_{n}$ is dense.

The proof is completed by observing that for each $x$ in $\cap_{n=1}^{\infty} G_{n}$ the set $E_{x}$ is a nowhere dense subset of $(0, \infty)$.

Although an uncountable union of sets of first category might occasionally produce a set of first category, it is surprising that under certain nonexotic conditions such an occurence will not be accidental. The conditions presented below appear in [2] and represent a refinement of a theorem of Banach [1].

BANAch CATEgory Theorem. Let $Y$ be a subspace of a topological space $X$ and let $\left\{V_{\lambda}: \lambda \in \Lambda\right\}$ be a collection of relatively open sets each of which is of first category in $X$ (though not necessarily in $Y$ ). Then $\cup_{\lambda \in \Lambda} V_{\lambda}$ is of first category in $X$.

We are now ready to expose the structure of closed sets in a normed linear space having points that see a residual subset.

Theorem 2. Let $C$ be a closed set of second category in a normed linear space $L$. Suppose that $C^{*}=\{x \in C: C-S(x)$ is of first catergory $\}$ is nonempty. Then

(a) $C^{*}$ is a closed convex set,

(b) $C=K \cup P$ where $K$ is a closed starshaped set with convex kernel $C^{*}$ and $P$ is of first category.

Proof. (a) We first show that $C^{*}$ is a closed set. Let $\left\{x_{n}\right\}$ be a sequence in $C^{*}$ convergent to a point $x$ (which must be in $C$ ). Clearly,

$$
C-S(x) \subset \bigcup_{n=1}^{\infty} \bigcap_{k=n}^{\infty}\left[C-S\left(x_{k}\right)\right] \text {. }
$$

Since $C-S\left(x_{k}\right)$ is of first category for each $k$ and the sets of first category form a $\sigma$-ideal, $C-S(x)$ is of first category. Hence, $x$ is in $C^{*}$. To establish the convexity of $C^{*}$, let $z$ and $y$ be distinct members of $C^{*}$. Since $z$ sees a set of second category via $C$, the corollary to Lemma 1 implies that $y$ sees $z$ via $C$. We have shown that $\operatorname{seg}[z, y] \subset C$, but it remains to show that $\operatorname{seg}[z, y] \subset C^{*}$.

Without loss of generality we can assume that $z=0$. By Theorem 1 the set $A$ of points $x$ of norm one such that $\{\alpha: \alpha>0$ and $\alpha x \in S(0)-S(y)\}$ is of second category in the line is of first category in the boundary of the unit ball. It easily follows that $A^{\prime}=\{\alpha x: \alpha>0$ and $x \in A\}$ is of first category in $L$. Now if $w$ is in $S(0)-A^{\prime}$, then $w$ cannot be in $S(y)^{\sim}$ because $\{\alpha: \alpha w /\|w\| \in S(0)-S(y)\}$ cannot contain an interval, a set of second category in the line. Hence, if $w$ is in $S(0)-A^{\prime}$, then $w$ is in $S(y)$. We have shown that $y$ sees every ray or line segment in $S(0)$ with one endpoint 0 in its entirety except for a subset of $S(0)$ of first category. Hence, each point of $\operatorname{seg}[0, y]$ has the same property. Since $S(0)-A^{\prime}$ is a residual subset of $C$, the convexity of $C^{*}$ is established.

(b) Since the relative complement of the star of each point in $C^{*}$ is relatively open and is of first category in $L$, the Banach Category Theorem implies that $K=\bigcap_{x \in C^{*}} S(x)$ is a closed residual subset of $C$. We now claim that each point 
of $K$ sees each point of $C^{*}$ via $K$, not merely via $C$. Fix $p$ in $K$ and $y$ in $C^{*}$. If $z$ is any other point of $C^{*}$, then since $\operatorname{seg}[y, z] \subset C^{*}$, it follows that $p$ sees $\operatorname{seg}[y, z]$ via $C$. Thus, the convex hull of $\{p, z, y\}$ is contained in $C$ so that $\operatorname{seg}[p, y] \subset S(z)$. Since $z$ was arbitrary, $\operatorname{seg}[p, y] \subset K$ and $K$ is starshaped with respect to $C^{*}$. By definition $C^{*}$ is contained in the convex kernel of $K$, and since $K$ is a residual subset of $C$, the reverse inclusion holds, too. We finally remark that Lemma 1 implies that the star of each point of $C-K$ is a set of first category. Hence, $C$ is the union of a closed starshaped set plus a set of insignificant size each point of which sees only a set of insignificant size.

We close by noting that the above decomposition theorem as a consequence of the Banach Category Theorem rests on the axiom of choice. However, a constructive proof can be obtained if $L$ is required to be separable: in this case $K$ can be taken to be the points mutually visible from a countable dense subset of $C^{*}$, or alternatively, one could provide a constructive proof of the Banach Category Theorem for second countable spaces.

\section{REFERENCES}

1. S. Banach, Theorème sur les ensembles de première categorie, Fund. Math. 16 (1930), 395-398.

2. K. Kuratowski, Topology, Vol. I. Academic Press, New York, 1966.

3. J. C. Oxtoby, Measure and category, Springer-Verlag, New York, 1971.

4. F. A. Valentine, Convex sets, McGraw-Hill, New York, 1964.

Department of Mathematics, California State University, Los Angeles, California 90032 